\title{
Catalytic role of boron atoms in self-interstitial clustering in $\mathrm{Si}$
}

\author{
Gyeong S. Hwang ${ }^{\text {a) }}$ and William A. Goddard III ${ }^{\text {b) }}$ \\ Materials and Process Simulation Center (139-74), California Institute of Technology, Pasadena, \\ California 91125
}

(Received 13 September 2002; accepted 30 May 2003)

\begin{abstract}
Using density functional theory (DFT) calculations and kinetic simulations, we have investigated the influence of boron atoms on self-interstitial clustering in Si. From DFT calculations of neutral interstitial clusters with a single $\mathrm{B}$ atom $\left(\mathrm{B}_{s} \mathrm{I}_{n}, n \leqslant 4\right)$, we find that the binding of $\mathrm{B}\left(\mathrm{B}_{s} \mathrm{I}_{n} \rightarrow \mathrm{I}_{n-1}\right.$ $\left.+\mathrm{B}_{s} \mathrm{I}\right)$ becomes substantially weaker than that of an interstitial $\left(\mathrm{B}_{s} \mathrm{I}_{n} \rightarrow \mathrm{B}_{s} \mathrm{I}_{n-1}+\mathrm{I}\right)$ when $n \geqslant 4$. This implies boron can be liberated while leaving an interstitial cluster behind. Our kinetic simulations including the boron liberation explain well experimental observations reported by J. L. Benton et al., J. Appl. Phys. 82, 120 (1997). (C) 2003 American Institute of Physics.
\end{abstract}

[DOI: $10.1063 / 1.1596729$ ]

Continued scaling of Si devices below $0.1 \mu \mathrm{m}$ requires formation of ultrashallow junctions to avoid short-channel effects. ${ }^{1}$ Ultralow-energy ion beams are currently most widely used to introduce dopants into the Si substrate. Generally this must be followed by high-temperature thermal annealing to eliminate substrate damage generated by energetic ion bombardment and to electrically activate the injected dopants. During the implantation and annealing, the dopant atoms exhibit transient enhanced diffusion (TED). This is a particularly serious problem for boron (a major p-type dopant), which exhibits large TED. The consequent doping profile spreading imposes a great difficulty in forming ultrashallow $p n$ junctions in deep submicron device structures.

It is now well understood that excess Si self-interstitials generated during implantation are mainly responsible for the B TED. Due to large mobility even at room temperature, ${ }^{2}$ single interstitials can diffuse and annihilate at the surface. Therefore, at the onset of annealing most interstitials are likely to remain in the form of clusters. It is widely believed that, in ultrashallow junction formation by ultralow energy ion implantation, the main sources for free interstitials during postimplantation annealing are small interstitial clusters and boron-interstitial complexes ${ }^{3-6}$ along with extended $\{311\}$ defects. The formation of boron-interstitial complexes have been extensively studied using first principles calculations, ${ }^{7,8}$ also supported by recent experiments. ${ }^{9}$ However, little is known about the role of single B atoms in the growth of small interstitial clusters.

Deep level transient spectroscopy recently provided direct evidence for the existence of small $\mathrm{Si}$ interstitial clusters. ${ }^{10}$ These results showed that the small interstitial clusters did not contain large numbers of B atoms even in a $\mathrm{B}$ doped layer (where $\mathrm{B}$ concentration was not high enough to induce B clustering). This was surprising because recent ab initio calculations ${ }^{7,8}$ suggested that $\mathrm{Si}$ interstitials interact very strongly with substitutional $\mathrm{B}$ to form stable $\mathrm{B}_{s} \mathrm{I}_{,} \mathrm{B}_{s} \mathrm{I}_{2}$,

\footnotetext{
${ }^{a)}$ Present address: Department of Chemical Engineering, The University of Texas, Austin, Texas.

b) Author to whom correspondence should be addressed; electronic mail: wag@wag.caltech.edu
}

and $\mathrm{B}_{s} \mathrm{I}_{3}$ complexes with large thermal stability comparable to that of small interstitial clusters. ${ }^{7,8}$

In order to provide a basis for understanding the formation of interstitial clusters in the presence of $\mathrm{B}$, we have carried out

(i) first-principles quantum mechanics calculations on the structures and energetics of small interstitial clusters with and without a single $\mathrm{B}$ atom and

(ii) kinetic model based simulations on boron-interstitial concentration variations.

These results suggest that B can catalyze the growth of small Si interstitial clusters.

Our first principles calculations are based on densityfunctional theory (DFT) within the local spin density approximation (LSDA), as implemented in the CPMD V3.3 package. ${ }^{11}$ We used a nonlocal, norm-conserving pseudopotential $^{12}$ and a plane-wave cutoff energy of 20 Ry. Defect systems considered in this study were modeled using 64-atom supercells with a fixed volume corresponding to a $\mathrm{Si}-\mathrm{Si}$ bond distance of $2.35 \AA$ in pure $\mathrm{Si}$. The atomic positions were allowed to relax fully until all residual forces were smaller than $5 \times 10^{-4}$ Hartree/Bohr. The geometries were optimized using only one $k$ point $(\Gamma)$ for the Brillouin-zone integrations. (The geometry optimization is rather insensitive to the number of $k$ point sets.) However, the total energies were reevaluated using a $(2 \times 2 \times 2)$ mesh of $k$ points in the scheme of Monkhorst-Pack. ${ }^{13}$

We first examined structure and energetics of a number of interstitial clusters containing only $\mathrm{Si}\left(\mathrm{I}_{n}, n \leqslant 3\right)$ and containing a single boron atom $\left(\mathrm{BI}_{n}, n \leqslant 4\right)$. Figure 1 shows the fully relaxed structure and LSDA energy for all clusters examined. To ensure that we would find the global minimum (instead of a high-energy local minimum state), we performed ab initio molecular dynamics followed by energy minimization for many different initial configurations.

The $\mathrm{B}_{s} \mathrm{I}$ configuration [Fig. 1(a)] has the Si interstitial (I) next to the $\mathrm{B}$ substitutional $\left(\mathrm{B}_{s}\right)$ in a tetrahedral site. The binding energy between $\mathrm{I}$ and $\mathrm{B}_{s}$ is calculated to be $1.14 \mathrm{eV}$ [virtually identical to $1.1 \pm 0.1 \mathrm{eV}$ obtained in a previous local density approximation (LDA) calculation]. ${ }^{15}$

For $\mathrm{B}_{s} \mathrm{I}_{2}$ we find the $\mathrm{B}$ to be substitutional with two 

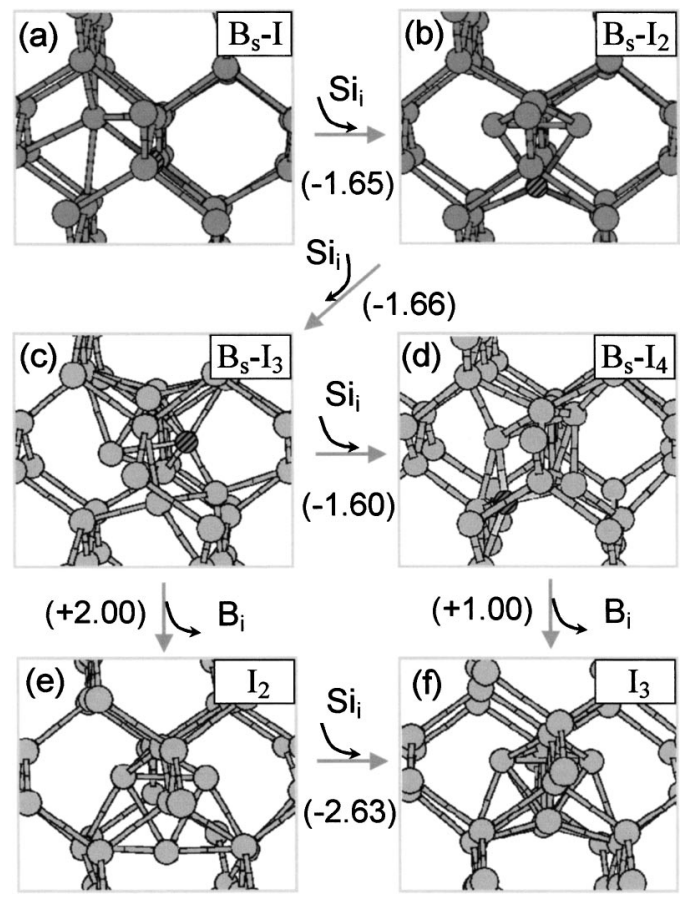

FIG. 1. Structure and energetics of boron-interstitial and interstitial only clusters examined in this study. Boron is indicated by the hatched ball.

interstitials on each side (dumbbell) in the $C_{1 h}$ symmetry [Fig. 1(b)]. We find the $\mathrm{B}_{s} \mathrm{I}_{2}$ cluster to be quite stable with a binding energy of $1.65 \mathrm{eV}$ (the energy required to release an interstitial from the $\mathrm{B}_{s} \mathrm{I}_{2}$ cluster). This is consistent with a recent LDA calculation which leads to a binding energy of $1.9 \mathrm{eV}$ between $\left(\mathrm{B}_{s} \mathrm{I}\right)^{+}$and $\mathrm{I}^{7-8}$

We also identified a stable neutral $\mathrm{B}_{s} \mathrm{I}_{3}$ structure [Fig. $1(\mathrm{c})]$ that appears quite different from the $\mathrm{BI}_{3}^{+}$configuration reported recently by Liu et al. ${ }^{7-8}$ Two Si lattice atoms (close to the B atom) are significantly displaced from their original position, allowing all bonds to be well reconstructed with appropriate bond lengths. We obtain a binding energy of 1.66 $\mathrm{eV}$ (energy required to release an interstitial from the $\mathrm{B}_{S} \mathrm{I}_{3}$ cluster).

Inserting an additional $\mathrm{Si}$ interstitial into the $\mathrm{B}_{s} \mathrm{I}_{3}$ cluster, leads to a $\mathrm{B}_{s} \mathrm{I}_{4}$ cluster that is also quite stable (a binding energy of $1.59 \mathrm{eV}$ ) toward releasing an interstitial. This $\mathrm{B}_{s} \mathrm{I}_{4}$ cluster [Fig. 1(d)] can be visualized as an $\mathrm{I}_{3}$ structure [see Fig. 1(f)] that is distorted by insertion of an interstitial boron.

This result suggests that the presence of B atoms may help the Si interstitials grow into compact configurations. For crystalline $\mathrm{Si}$, the compact tri-interstitial cluster $\left(\mathrm{I}_{3}\right)$ has a formation energy $\sim 0.2 \mathrm{eV}$ lower than the ring cluster and 0.4 $\mathrm{eV}$ lower than elongated clusters. ${ }^{16}$

Figure 1 also shows compact $\mathrm{I}_{2}$ and $\mathrm{I}_{3}$ clusters (fully relaxed structures). The $\mathrm{I}_{2}$ and $\mathrm{I}_{3}$ formation energies are calculated to be 2.63 and $1.99 \mathrm{eV}$, respectively (in excellent agreement with the values of 2.5 and $1.9 \mathrm{eV}$ obtained in recent DFT-LDA calculations). ${ }^{16}$ We calculate the formation energy for a Si self-interstitial to be $3.3 \mathrm{eV}$, very close to the result of 3.3-3.35 eV from other DFT-LDA calculations. ${ }^{15}$

We also examined the possibility of boron liberation from the B-containing interstitial clusters. Our DFT-LSDA calculations show that the decay of $\mathrm{B}_{s} \mathrm{I}_{3}$ into $\mathrm{B}_{s} \mathrm{I}$ and $\mathrm{I}_{2}$ requires an energy of $2.0 \mathrm{eV}$, which is larger than the $1.66 \mathrm{eV}$ Downloaded 21 Dec 2005 to 131.215.225.171. Redistribution subje for $\mathrm{B}_{s} \mathrm{I}_{3} \rightarrow \mathrm{B}_{s} \mathrm{I}_{2}+\mathrm{I}$. [The barrier for boron-interstitial pair diffusion $(0.68 \mathrm{eV})^{17}$ is also likely to be larger than that for interstitial diffusion $(0.15-0.2 \mathrm{eV}).]^{18}$ Thus from $\mathrm{B}_{s} \mathrm{I}_{3}$ we calculate that boron $\left(\mathrm{B}_{s} \mathrm{I}\right)$ liberation is less favorable than interstitial liberation.

However, for $\mathrm{B}_{s} \mathrm{I}_{4}$, the binding energy of boron $\left(\mathrm{B}_{s} \mathrm{I}_{4}\right.$ $\rightarrow \mathrm{B}_{s} \mathrm{I}+\mathrm{I}_{3}$ ) decreases significantly to $1.0 \mathrm{eV}$ while the interstitial binding energy $\left(\mathrm{B}_{s} \mathrm{I}_{4} \rightarrow \mathrm{I}+\mathrm{B}_{s} \mathrm{I}_{3}\right)$ of $1.60 \mathrm{eV}$ remains nearly unchanged. These results demonstrate that the binding energy of boron decreases as the number of integrated interstitials increases. This is consistent with the very weak interaction between boron and extended $\{311\}$ defects. ${ }^{7}$ This binding energy reduction would allow boron to release more favorably than an interstitial from $\mathrm{B}_{s} \mathrm{I}_{n}$ when $n \geqslant 4$.

These results suggest that boron atoms may catalyze interstitial agglomeration. That is, boron atoms provide a means to capture and integrate interstitials into a cluster, followed by the emission of the boron to facilitate formation of additional interstitial clusters. In this study, we consider only charge neutral clusters, which may lead to some errors in estimating the binding energies of boron-interstitial clusters (as the cluster structures and energies could change significantly at different charge states) ${ }^{7,8}$ However, we believe that our results give a reasonable description in the overall physical picture, particularly for boron-interstitial clusters under near intrinsic conditions (as considered herein). In fact, current first principles calculations on various charge-state systems are still quite uncertain. ${ }^{7,8,14}$

This catalytic reaction may provide an explanation for the experimental observation that a large fraction of B atoms remains electrically active. (In these experiments, the $\mathrm{Si}$ implant doses were $1 \times 10^{9}-5 \times 10^{13} \mathrm{~cm}^{-2}$ and the doped B concentration was about $7 \times 10^{15} \mathrm{~cm}^{-3}$.)

To illustrate this process we carried out kinetic-model based simulations in which we started with a boron concentration of $7 \times 10^{15} \mathrm{~cm}^{-3}$ and added interstitials at a rate of $10^{16} \mathrm{~cm}^{-3} \mathrm{~s}$ for $10 \mathrm{~s}$ to achieve a total concentration of $10^{17} \mathrm{~cm}^{-3}$. The interstitial diffusivity is approximated using $0.001 \times \exp \left(-E_{m} / k_{B} T\right)$, where $E_{m}=0.2 \mathrm{eV} .{ }^{18}$ The calculated diffusivity of $3 \times 10^{-7} \mathrm{~cm}^{2} / \mathrm{s}$ at room temperature is in good agreement with an experimental estimate of $\geqslant 10^{-7} \mathrm{~cm}^{2} / \mathrm{s}$. ${ }^{19}$

Figure 2(a) shows the concentrations of boron-interstitial clusters and self-interstitial clusters after $10 \mathrm{~s}$ of interstitial implantation into the boron-doped layer at room temperature. Here, boron clustering can be ignored due to the low B concentration. Our simulation result shows that about $97 \%$ of the injected interstitials are incorporated into pre-existing boron sites with just 3\% remaining as interstitial clusters. This ratio is a function of the interstitial injection rate and diffusivity; that is, a higher fraction of interstitials is aggregated into boron-interstitial clusters as the injection rate decreases and/or the diffusivity increases. Although many details of defect dynamics (such as vacancy formation) are ignored in our simulation, we see that boron can efficiently capture implanted interstitials to form boron-interstitial complexes during implantation. We expect that this interstitial aggregation will lead to entrenchment of most boron atoms (if the implanted interstitial concentration is far greater than the boron concentration). Indeed our simulation results show that (virtually) all boron atoms are embedded in interstitial clusters. to AIP license or copyright, see http://apl.aip.org/apl/copyright.jsp 


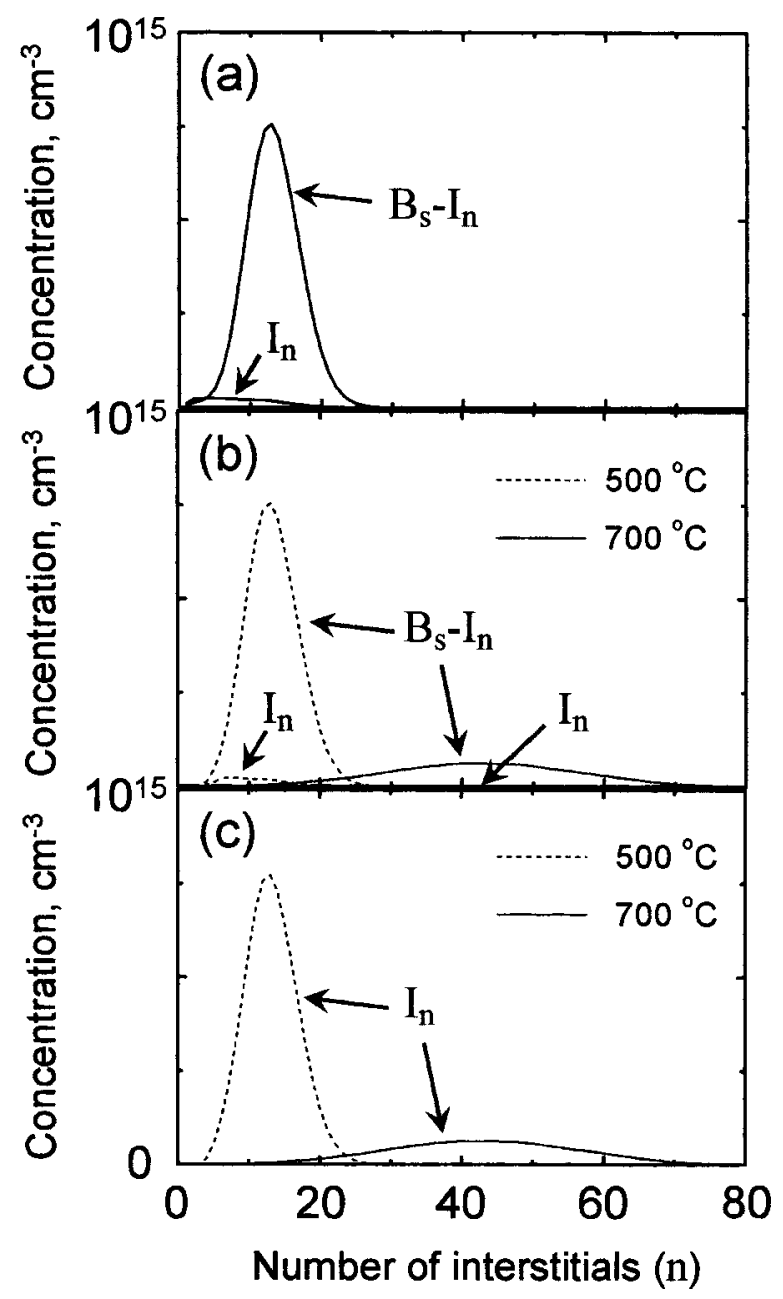

FIG. 2. The concentrations of boron-interstitial $\left(\mathrm{B}_{s}-\mathrm{I}_{n}\right)$ and self-interstitial $\left(\mathrm{I}_{n}\right)$ clusters. (a) After $10 \mathrm{~s}$ of interstitial implantation, (b)-(c) after $10 \mathrm{~min}$ of subsequent postimplantation annealing at 500 and $700{ }^{\circ} \mathrm{C}$ [(b) without and (c) with boron liberation from boron-interstitial clusters].

Figure 2 also shows simulated concentrations of boroninterstitial and self-interstitial clusters after $10 \mathrm{~min}$ of subsequent postimplantation annealing at 500 and $700{ }^{\circ} \mathrm{C}$ (with [Fig. 2(c)] and without [Fig. 2(b)] boron liberation from boron-interstitial complexes). In these simulations, we assume that the energy barriers for interstitial release from boron-interstitial and interstitial clusters are comparable [approximated as the sum of $E_{m}$ and $E_{b} ; E_{m}=0.2 \mathrm{eV}^{18}$ and $E_{b}\left(n_{\mathrm{I}}\right)=2.7-2.65\left[n_{\mathrm{I}}^{1 / 2}-\left(n_{\mathrm{I}}-1\right)^{1 / 2}\right] \mathrm{eV},{ }^{20}$ where $n_{\mathrm{I}}$ is the number of interstitial incorporated]. Without boron liberation about $100 \%$ of boron atoms are still embedded in interstitial clusters at $500{ }^{\circ} \mathrm{C}$ and $\sim 30 \%$ are at $700{ }^{\circ} \mathrm{C}$. (The fraction of substitutional boron decreases with the concentration of injected interstitials.) On the other hand, when we allow boron to release (once the boron-interstitial cluster size becomes greater than $\mathrm{B}_{s}-\mathrm{I}_{4}$ ), virtually all boron atoms recover to substitutional locations. Thus, our simulations demonstrate clearly that without boron liberation, a significant amount of boron would still remain as boron-interstitial clusters (under the conditions considered in this study).

Now we suspect that the boron-catalyzed reaction outlined earlier may facilitate the formation of small interstitial clusters, especially during implantation and/or early stages of post-implantation annealing. As a result, a larger fraction of interstitials would remain in the substrate. Since B-TED is Downloaded 21 Dec 2005 to 131.215.225.171. Redistribution subject mainly determined by the availability of interstitials, the increased concentration of interstitials would influence doping profile evolution. This suggests the importance of including the B-catalyzed process, along with interstitial clustering and boron-interstitial complex formation/dissolution, in modeling ultrashallow junction formation.

In summary, based on our first principles calculations and kinetic simulations we propose and validate the mechanism of boron-catalyzed interstitial cluster formation. Our first principles calculations show the reduction of boron and interstitial interaction with the number of interstitials incorporated, leading to boron liberation while leaving an interstitial cluster. A comparison of experiments with kinetic simulations supports the boron liberation. The liberated boron may then induce formation of additional interstitial clusters, i.e., B-catalyzed interstitial cluster formation.

The authors thank the Seiko-Epson Corporation for providing financial support and the authors thank Masamitsu Uehara and Yuzuru Sato of Seiko-Epson for many helpful discussions. G.S.H. also thanks the Welch Foundation for their partial financial support. The facilities of the MSC used in this work are supported by grants from DOE-ASCI, ARO/DURIP, ONR/DURIP, ARO/MURI, ONR/MURI, IBM, NIH, NSF, ChevronTexaco, Beckman Institute, General Motors, and Asahi Kasei.

${ }^{1}$ E. C. Jones and E. Ishida, Mater. Sci. Eng., R. 24, (1998), and references cited therein.

${ }^{2}$ G. D. Watkins, Phys. Rev. B 12, 5824 (1975).

${ }^{3}$ L. H. Zhang, K. S. Jones, P. H. Chi, and D. S. Simons, Appl. Phys. Lett. 67, 2025 (1995).

${ }^{4}$ A. D. Lilak, S. K. Earles, M. E. Law, and K. S. Jones, Appl. Phys. Lett. 74, 2038 (1999).

${ }^{5}$ E. Schroer, V. Privitera, F. Priolo, E. Napolitani, and A. Carnera, Appl. Phys. Lett. 74, 3996 (1999).

${ }^{6}$ G. Mannino, N. E. B. Cowern, F. Roozeboom, and J. G. M. van Berkum, Appl. Phys. Lett. 76, 855 (2000).

${ }^{7}$ X. Y. Liu, W. Windl, and M. P. Masquelier, Appl. Phys. Lett. 77, 2018 (2000).

${ }^{8}$ T. J. Lenosky, B. Sadigh, S. K. Theiss, M. J. Caturla, and T. D. de la Rubia, Appl. Phys. Lett. 77, 1834 (2000).

${ }^{9}$ A. Agarwal, H. J. Gossmann, D. J. Eaglesham, S. B. Herner, A. T. Fiory, and T. E. Haynes, Appl. Phys. Lett. 74, 2331 (1999).

${ }^{10}$ J. L. Benton, S. Libertino, P. Kringhøj, D. J. Eaglesham, J. M. Poate, and S. Coffa, J. Appl. Phys. 82, 120 (1997); J. L. Benton, K. Halliburton, S. Libertino, D. J. Eaglesham, and S. J. Coffa, Appl. Phys. Lett. 84, 4749 (1998).

${ }^{11}$ J. Hutter, A. Alavi, T. Deutch, M. Bernasconi, S. Goedecker, D. Marx, M. Tuckerman, and M. Parrinello, CPMD, MPI fuer Festkoerperforschung and IBM Zurich Research Laboratory 1995-1999.

${ }^{12}$ S. Goedecker, M. Teter, and J. Hutter, Phys. Rev. B 54, 1703 (1996).

${ }^{13}$ H. J. Monkhorst and J. D. Pack, Phys. Rev. B 13, 5188 (1976).

${ }^{14}$ W. Windl, M. M. Bunea, R. Stumpf, S. T. Dunham, and M. P. Masquelier, Phys. Rev. Lett. 83, 4345 (1999).

${ }^{15}$ J. Zhu, T. D. de la Rubia, L. H. Yang, C. Maihiot, and G. H. Gilmer, Phys. Rev. B 54, 4741 (1996), and references cited therein.

${ }^{16}$ J. Kim, F. Kirchhoff, J. W. Wilkins, and F. S. Khan, Phys. Rev. Lett. 84, 503 (2000).

${ }^{17}$ B. Sadigh, T. J. Lenosky, S. K. Theiss, M.-J. Caturla, T. D. de la Rubia, and M. A. Foad, Phys. Rev. Lett. 83, 4341 (1999).

${ }^{18}$ W. K. Leung, R. J. Needs, G. Rajagopal, S. Itoh, and S. Ihara, Phys. Rev. Lett. 83, 2351 (1998).

${ }^{19}$ E. J. H. Collart, K. Weemers, N. E. B. Cowern, J. Politiek, P. H. L. Bancken, J. G. M. von Berkum, and D. J. Gravesteijn, Nucl. Instrum. Methods Phys. Res. B 139, 98 (1998).

${ }^{20}$ L. Pelaz, G. H. Gilmer, M. Jaraiz, S. B. Herner, H. J. Gossmann, D. J. Eaglesham, G. Hobler, C. S. Rafferty, and J. Barbolla, Appl. Phys. Lett. 70, 2285 (1997). 